\title{
On the Applicability of Similarity Theory for the Stable Atmospheric Boundary Layer over Complex Terrain
}

\section{Barskov, K. V.}

2018-09

Barskov , K V , Glazunov , A V , Repina , I A , Stepanenko , V M , Lykossov , V N \& Mammarella , I 2018 , ' On the Applicability of Similarity Theory for the Stable Atmospheric Boundary Layer over Complex Terrain ' , Izvestiya. Atmospheric and oceanic physics, vol. 54 , no. 5 , pp. 462-471 . https://doi.org/10.1134/S0001433818050031

http://hdl.handle.net/10138/288217

https://doi.org/10.1134/S0001433818050031

Downloaded from Helda, University of Helsinki institutional repository.

This is an electronic reprint of the original article.

This reprint may differ from the original in pagination and typographic detail.

Please cite the original version. 


\title{
On the Applicability of Similarity Theory for the Stable Atmospheric Boundary Layer over Complex Terrain
}

\author{
K. V. Barskov ${ }^{a, b}$, A. V. Glazunov ${ }^{b, c}$, I. A. Repina ${ }^{a, b, *}$, V. M. Stepanenko ${ }^{b, e}$, \\ V. N. Lykossov ${ }^{b, c}$, and I. Mammarella ${ }^{d}$ \\ ${ }^{a}$ Obukhov Institute of Atmospheric Physics, Russian Academy of Sciences, Moscow, 119017 Russia \\ ${ }^{b}$ Research Computing Center, Moscow State University, Moscow, 119234 Russia \\ ${ }^{c}$ Marchuk Institute of Numerical Mathematics, Russian Academy of Sciences, Moscow, 119991 Russia \\ ${ }^{d}$ Institute for Atmospheric and Earth System Research/Physics, Faculty of Science, University of Helsinki, \\ Helsinki, 00014 Finland \\ ${ }^{e}$ Faculty of Geography, Moscow State University, Moscow, 119234 Russia \\ *e-mail:repina@ifaran.ru \\ Received August 21, 2017; in final form, November 28, 2017
}

\begin{abstract}
Micrometeorological measurements in the atmospheric boundary layer over a hilly forest terrain have been made on a meteorological tower at several levels from the forest canopy top to a height that exceeds the height of trees almost seven times. A semiempirical length scale depending on the local topography features and the underlying surface type has been proposed and calculated. This scale has been shown to allow the universal functions of the Monin-Obukhov similarity theory to be corrected for a stable atmospheric boundary layer over complex terrain without substantial modification when compared to the universal functions over a homogeneous surface with small roughness elements. This approach can be used to refine the methods for calculating turbulent momentum fluxes from profile measurements over spatially inhomogeneous landscapes.
\end{abstract}

Keywords: atmospheric boundary layer, complex terrain, stable stratification, Monin-Obukhov similarity theory, universal functions, eddy-covariance measurements

DOI: $10.1134 / \mathrm{S} 0001433818050031$

\section{INTRODUCTION}

Present-day methods for determining the parameters of turbulent exchange between the Earth's surface and the atmosphere use theories that hypothesize stationarity and homogeneity. The traditional parametrizations in atmospheric modeling and energy-balance and biogeochemical calculations are based on the Monin-Obukhov similarity theory (MOST) [1], which assumes an underlying surface with a uniform horizontal distribution of aerodynamic and temperature roughness and turbulent flows [2]. The MOST has been experimentally verified for homogeneous and quasi-homogeneous surfaces since its introduction, which can be confirmed by hundreds of relevant references [for example, 3-9]. Significantly fewer experimental data have been collected over complex terrains. The conditions of statistical stationarity and uniformity are broken for inhomogeneous landscapes and surfaces with large roughness elements. The assumption that the vertical turbulent fluxes of heat, moisture, and momentum are height-invariant is likewise broken [10, 11].

There has been increased recent interest in the study of energy and mass exchange over the forest can- opy. This is largely associated with the study of the biogeochemical cycle, i.e., the processes of momentum, heat, and matter exchange between the atmosphere and vegetation [12]. In view of the strong dependence of heat, carbon dioxide, and water-vapor transfer on environmental conditions; the species composition and structure of vegetation; and the biophysical and biochemical processes occurring in plants and soil, one can regard the turbulent exchange as an indicator of the state, development, and growth of plants [13]. Studies of the structure of atmospheric turbulence in forests and over forest canopy are needed not only for analyzing the biospheric processes and using them adequately in models of global climate changes [14], but also for calculating the transport of aerosols and gaseous admixtures [15], forecasting adverse weather phenomena [16], developing analytical and numerical models of the atmospheric boundary layer dynamics [6], and advancing windpower engineering [17].

Experimental studies have shown that, due to the absence of a constant flux layer over the forest, the traditional flux-gradient relationships cannot be used [18]. The motions associated with organized turbulence play an important role in turbulent exchange both 
inside and above the forest [19]: emerging coherent structures generate an additional contribution of energy to the flux spectra and cospectra in the surface layer [20]. The stable boundary layers typical for nighttime hours in tropical and middle latitudes and for winter in polar and circumpolar regions are characterized by the formation of coherent structures with a significant effect on turbulent transfer. The turbulence over the forest under stable stratification was discussed, for example, in [21-23]; however, these studies considered mostly nighttime boundary layers. There have been almost no studies for boreal forests with long-lived stable boundary layers during the winter period.

The studies addressing the theory of turbulent transfer inside and above the forest were largely focused on the homogeneous vegetation cover on a flat surface [24-27]. In nonideal, but more realistic, conditions (such as a forest with lakes and meadows, nonuniform tree height and density, complex terrain, and patchy vegetation), the exchange processes become even more complex [28]. The influence of the vegetation cover inhomogeneity on turbulent exchange and turbulent motion is mostly analyzed using approaches that are based on analytical and numerical modeling. For example, an analytical model of wind flow inside and above the vegetation cover with a weakly varying vegetation density was developed in [29].

Our study uses data from micrometeorological measurements at the meteorological tower of the SMEAR II station (Hyytiälä, Southern Finland) [30]. The terrain near the station includes low hills covered with coniferous forest. The measurements were made at several levels: the first was located near the topmost of trees and the higher level was located at a height that was slightly lower than the stable boundary layer height under given conditions. The main objective of this paper is to find the regularities of turbulent momentum exchange under stable stratification over complex terrain in terms of the similarity theory.

\section{STABLE ATMOSPHERIC BOUNDARY LAYER OVER UNDERLYING SURFACE WITH LARGE ROUGHNESS ELEMENTS}

Due to self-similarity and a relatively small effect of molecular viscosity and thermal conductivity on the thermodynamics of turbulent flow in a horizontally homogeneous surface layer, there are universal dependences of dimensionless statistical characteristics of stratified turbulence on the stability parameter $\zeta=\kappa z / L$. Here, $z$ is the height; $L=U_{*}^{3} / F_{b}$ is the Obukhov length scale, which depends on dynamic velocity $U_{*}$ and buoyancy flux near the surface; and $\kappa=0.4$ is von Kármán's constant. The Monin-Obukhov similarity theory involves semiempirical functions that associate the momentum, heat, moisture and any other passive scalar fluxes with vertical gradients of respective averaged quantities. These universal functions are normally used to calculate the turbulent surface flows in atmospheric models with the help of the gradient or profile method that requires data only on the average wind speed and other scalars measured at two or more levels.

For a weakly stable $(L>z)$ and moderately stable stratification $(L \sim z)$, the dimensionless gradient of mean wind speed $\Phi_{m}(\zeta)$ is approximated by the linear relationship [31]

$$
\Phi_{m}(\zeta)=\frac{d\langle U\rangle}{d z} \frac{\kappa z}{U_{*}} \approx 1+C_{m} \zeta,
$$

where $\langle U\rangle$ is the upstream mean wind speed and $C_{m} \approx 5$ is an empirical coefficient.

The MOST assumes that the roughness layer thickness is negligibly small in comparison with the measurement height $z$ and the turbulent fluxes in the surface layer are constant with respect to height. In an equilibrium stable atmospheric boundary layer (ABL) of thickness $H \sim 10^{1}-10^{2} \mathrm{~m}$, this approximation is valid only at the Earth's surface, because the turbulent fluxes in the presence of the Coriolis force are distributed in height as follows [32]:

$$
\begin{aligned}
& |\tau(z)| / U_{*}^{2} \approx(1-z / H)^{3 / 2}, \\
& F_{b}(z) /\left.F_{b}\right|_{z=0} \approx(1-z / H) .
\end{aligned}
$$

Here, $\tau(z)=\left(\left\langle u^{\prime} w^{\prime}\right\rangle,\left\langle v^{\prime} w^{\prime}\right\rangle\right)$ is the vertical turbulent momentum flux normalized to air density, $F_{b}(z)=\left\langle b^{\prime} w^{\prime}\right\rangle$ is the buoyancy flux associated with temperature fluctuations $T$ under given conditions (at low air humidity and near the surface) as $F_{b} \approx\left\langle T^{\prime} w^{\prime}\right\rangle \frac{g}{T_{0}}, T_{0}$ is the average air temperature, and the angle brackets mean statistical averaging (in our case, ergodicity was assumed, and the measurement data were averaged over 30-min time intervals).

It is empirically found (see, for example, [33]) that, without any loss of accuracy, one can replace the surface friction velocity and the buoyancy surface flux in formula (1) by their values at the measurement level. In addition, the aerodynamic properties of surfaces with large roughness elements are commonly characterized by two parameters with the dimension of length: the roughness parameter $z_{0}$ and the displacement height $D$. A widespread approximation for the flux-gradient relationship in a stable ABL over this kind of surface (for example, over forest canopy or urban surface) has the form [34]

$$
\begin{gathered}
\phi_{m}(\xi)=\frac{d\langle U\rangle}{d z} \frac{\kappa(z-D)}{u_{*}} \approx a(\gamma)+C_{m} \xi, \\
\xi=\frac{\kappa(z-D)}{\Lambda},
\end{gathered}
$$

where $\gamma=(z-D) / H, a(\gamma)=\mathrm{const} \approx 1$, and $u_{*}=|\tau|^{1 / 2}$ and are the dynamic velocity and Obukhov length 
scale at the measurement height $z$, respectively (by contrast, and $L$ denote the same quantities near the surface). Relation (3) is valid in a larger height range (up to almost half the ABL height) than the original formula of (1) of the MOST and does not depend on the type of turbulent flux profiles caused by a large-scale height-distributed external dynamic influence [35].

Formula (3) cannot be used under conditions in the immediate vicinity of roughness elements if the vertical scale of topographic inhomogeneities is comparable with $(z-D)$ and/or if the layer of roughness elements is arranged so that the structure of turbulence above it differs significantly from that in a shear flow over a flat wall. The latter case can be exemplified by turbulence over sparse vegetation, which, according to numerical calculations [36] and measurement data [37], shows a tendency for the formation of large coherent vortices; in turn, this leads to a specific form of the observed flux-gradient relationships differing from the standard relationships of the similarity theory: $a(\gamma) \neq$ const $>1$ (see, for example, the laboratory measurements described in [34]).

For the values of the stability parameter $\xi>2-3$, the dimensionless gradient $\phi_{m}$ usually decreases in comparison with the values calculated from formula (3) (see, for example, [33]). A spectral analysis of the results of an LES simulation of a stable ABL over an urban-type surface indicated that this decrease is caused by large eddies of the scale of the total boundary layer thickness [38]. The relative contribution of these eddies to the vertical momentum transfer is maximal in the upper ABL, where they provide a more efficient mixing than the locally generated shear turbulence. Here, we use the conclusions drawn in [38] to explain data for the highest measurement level, which is mostly located in the upper ABL.

There is a practical need to interpret and assimilate meteorological and micrometeorological observations at towers surrounded by a natural spatially inhomogeneous landscape with a unique topography and vegetation cover. It is not easy to find generalizing regularities for these measurements and introduce analytical corrections to the MOST on their basis; this is beyond the scope of our study. On the contrary, we consider the results of micrometeorological measurements in a given landscape that cannot be directly generalized. We show the possibility of introducing a correction to functions of type (3) from the conditions of the given landscape. This method is of practical interest when the direct pulsation measurements of turbulent fluxes are rare and cannot cover the whole range of possible stratifications.

\section{THE HYPOTHESIS}

The data are analyzed using an assumption similar to A.M. Obukhov's hypothesis that the turbulence scale (or Prandtl's mixing length) $l_{t}$ in the steady-state turbulent flow depends only on the local geometry and is independent of the dynamics of the flow itself [39]. Following this hypothesis, Obukhov used conformal transformations of the cross section of an infinitely extended domain onto the half-plane to obtain turbulent length scales $l_{t}$ and average velocities $\langle u\rangle$ in neutrally stratified flows between two parallel plates and in square and circular cross-section tubes, which are consistent with measurement data.

We assume that the vegetation and topography specify a unique geometry that determines the base scale of turbulence $l_{t}^{b}$ under neutral stratification. This scale cannot be expressed analytically, but it specifies a flux-gradient relationship and can be calculated from measurement data $\xi_{b}<\xi<\xi_{s}$ as follows:

$$
l_{t}^{b}=\left\langle u_{*} / \frac{d\langle U\rangle}{d z}\right\rangle=\kappa(z-D) / a(\gamma) .
$$

Here, $\xi_{b}$ and $\xi_{s}$ are some small thresholds $\left(\xi_{b}<0\right.$, $\left.\xi_{s}>0,\left|\xi_{b}\right| \ll 1\right)$ bounding the range of ABL parameters, where the stratification effect on turbulence is small; the outer angle brackets denote averaging over the whole interval $\left[\xi_{b}, \xi_{s}\right]$.

Using (for normalization) the scale $l_{t}^{b}(z)$ instead of the scales that depend on the distance from the surface $l_{t}=\kappa z$ or $l_{t}=\kappa(z-D)$ and used in the classical theory of a logarithmic surface layer, we assume that the stratification effects manifest themselves universally, independent of the local geometry, which is taken into account only through the scale $l_{t}^{b}(z)$. Then, the modified dimensionless velocity gradient in the surface layer over the forest

$$
\phi_{m}^{\prime}\left(\xi^{\prime}\right)=\frac{d\langle U\rangle}{d z} \frac{l_{t}^{b}}{u_{*}}
$$

like the dimensionless gradients $\Phi_{m}(\zeta)$ and $\phi_{m}(\xi)$ (see relationships (1) and (3), which hold over a flat surface), can be approximated by the linear function

$$
\phi_{m}^{\prime}\left(\xi^{\prime}\right) \approx 1+C_{m}^{\prime} \xi^{\prime}
$$

with the same value of the coefficient $C_{m}^{\prime}=C_{m} \approx 5$ in a wide range of values of the new stability parameter $\xi^{\prime}=l_{t}^{b} / \Lambda$.

It should be noted that formulas (3) and (6) in terms of turbulence scales can be written as

$$
\frac{1}{l_{t}} \approx \frac{1}{\kappa(z-D)}+\frac{C_{m}}{L \Lambda}, \frac{1}{l_{t}} \approx \frac{1}{l_{t}^{b}}+\frac{C_{m}^{\prime}}{\Lambda} .
$$

The terms associated with the local geometry of stratification are independent terms in expressions (7). Our hypothesis is reduced to the supposition that $C_{m}^{\prime}$ for turbulent flows over different surface types (including a statistically homogeneous surface with 
small roughness elements) is not a function of the parameters that govern the aerodynamic properties of these surfaces and remains constant in the lower part of the stable ABL.

\section{CORRECTION OF THE GRADIENT OF MEAN VELOCITY NEAR THE UPPER ABL LAYER}

Another factor complicating the analysis and interpretation of measurement data at upper levels is the proximity of these levels to the upper ABL boundary, where, as was noted in the Introduction, local scaling is not justified due to the presence of large eddies. Here, the ABL height varies for different stratifications and the dependence of the base scale $l_{t}^{b}$ on it cannot be determined by the method described above.

Let the dimensionless gradient $\Phi_{m}$ under neutral stratification in the ABL of any height $H$ be close to the averaged dimensionless velocity gradient calculated from the ensemble of measurement data with neutral stratification. Let us introduce an average length scale that is similar to $\mathrm{K} z$ in formula (1) but takes into account the effect of topography and vegetation cover:

$$
l_{t}^{b}=\left\langle U_{*} / \frac{d\langle U\rangle}{d z}\right\rangle
$$

where the outer angle brackets denote averaging over a sample of data with neutral stratification.

Then, the base scale of turbulence (the scale induced by topography, vegetation, and the limited height of the surface layer) can be approximated as

$$
l_{t}^{n b} \approx l_{t}^{b}|\tau|^{1 / 2} / U_{*},
$$

where $U_{*}$ is the dynamic velocity at the height of the measurement level $z_{1}$ that is closest to the surface.

We generate the following length scales

$$
l_{t}^{g}=\frac{l_{t}^{n b}}{1+C_{m} l_{t}^{B} / L}, \quad l_{t}^{s}=\frac{l_{t}^{n b}}{1+C_{m} l_{t}^{n b} / \Lambda} .
$$

The scale $l_{t}^{g}$ is associated with surface fluxes, includes a correction for the effect of topography and vegetation cover, and is responsible for large eddies with dimensions that are comparable to the height $z$.

The scale $l_{t}^{s}$ takes the local values of buoyancy and momentum fluxes into account, is associated with small-scale shear turbulence generated at the height of measurements, and includes also the correction associated with the unique landscape structure. Without buoyancy forces, both scales are equal to the scale $l_{t}^{n b}$, which allows the dimensionless gradient $\Phi_{m}$ to be close to the gradient averaged over a sample of data with neutral stratification. It follows from (10) that the scales in a steady-state equilibrium stable $\mathrm{ABL}$ are related as $l_{t}^{s} \leq l_{t}^{g}$.

Since buoyancy flux (2) in this ABL is linear in the absence of internal heat and moisture sources distributed in its depth, we can approximate the introduced length scales as

$$
l_{t}^{g} \approx \frac{l_{t}^{B}|\tau|^{1 / 2} / U_{*}}{1+C_{m} l_{t}^{B} / L}, \quad l_{t}^{s} \approx \frac{l_{t}^{B}|\tau|^{1 / 2} / U_{*}}{1+\left(U_{*}^{2}(1-\sigma)|\tau|\right) C_{m} l_{t}^{B} / L},
$$

where $\sigma=\frac{z-z_{1}}{H-z_{1}}$ is the dimensionless height. Here, the introduced length scales do not explicitly depend on the Obukhov length scale away from the surface $\Lambda(z)$. We can further approximate using power-law dependence (2) of the momentum flux magnitude on height:

$$
l_{t}^{g} \approx \frac{l_{t}^{B}(1-\sigma)^{3 / 4}}{1+C_{m} l_{t}^{B} / L}, \quad l_{t}^{s} \approx \frac{l_{t}^{B}(1-\sigma)^{3 / 4}}{1+(1-\sigma)^{-1 / 2} C_{m} l_{t}^{B} / L} .
$$

The results of numerical experiments using an LES model [40] revealed that $l_{t} \approx c k_{t}^{-1}$ with good accuracy, where $c$ is a constant, and $k_{t}=\int k C_{u w} d k / \int C_{u w} d k$ is the weighted-mean wavenumber with respect to the cospectrum $C_{u w}$. Given the processes contributing to the turbulent momentum flux are multiscale, it makes sense to approximate the total turbulent scale $l_{t}$ by interpolating several length scales with respective weight coefficients (an approximation of the abovementioned integral).

We use this technique to obtain the final expression for the turbulence scale:

$$
l_{t}^{m i x}=\left(C_{1} \frac{1}{l_{t}^{g}}+C_{2} \frac{1}{l_{t}^{s}}\right)^{-1}=\frac{C l_{t}^{g} l_{t}^{s}}{l_{t}^{g}+(C-1) l_{t}^{s}} .
$$

Only one constant $C$ is left in formula (13), because the required scale near the surface must coincide with the scale $l_{t}^{b}$, which takes into account only the surface properties not depending on the stratification and ABL height.

The study [38] obtained $C \approx 5$ and showed that the approximation similar to formula (13) (except the corrections introduced here for the effect of topography and vegetation) gives the flux-gradient relationship $l_{t}^{\text {mix }}(d\langle U\rangle / d z) \approx \mid \tau^{1 / 2}$ for different external influences supporting quasi-equilibrium turbulence in a stable ABL.

Now, we check the assumptions on the measurement data available from the SMEAR II station. If the assumptions are valid, the following results should be obtained:

$$
\phi_{m}^{\prime \prime}=\frac{d\langle U\rangle}{d z} \frac{l_{t}^{m i x}}{u_{*}} \approx 1
$$




$$
\phi_{m}^{\prime}\left(\xi^{\prime}, \sigma\right)=\frac{d\langle U\rangle}{d z} \frac{l_{t}^{b}}{u_{*}}=\frac{l_{t}^{b}}{l_{t}^{m i x}}
$$

Here, $l_{t}^{b}$ is the base turbulence scale and $l_{t}^{\text {mix }}$ is the turbulence scale that takes the ABL stratification and height into account.

It should be noted that now the function $\phi_{m}^{\prime}\left(\xi^{\prime}\right)$ is already nonuniversal and depends on both the stability parameter $\xi^{\prime}=l_{t}^{b} / \Lambda$ and the dimensionless height $\sigma=\frac{z-z_{1}}{H-z_{1}}$. In the lower $\mathrm{ABL}$, this dependence is weak: at lower levels, the gradients calculated by formulas (15) and (6) must coincide. For large values of $\xi$ typical for the upper ABL, formula (15) yields scattered gradients $\phi_{m}^{\prime}$ depending on $\sigma$ and its decreased values in comparison with the values calculated from formula (6). It is this behavior of the dimensionless velocity gradient that can be usually observed for locally scaled observation data [33].

\section{PRELIMINARY CHOICE OF OBSERVATION DATA}

We used monitoring data on the characteristics of atmospheric turbulence at different levels of the 127-m tower located at the SMEAR II research station (Station for Measuring Ecosystem-Atmosphere Relations, Hyytiälä, Finland, $24^{\circ} 17^{\prime} 13^{\prime \prime}$ E, 61 $51^{\circ} 5^{\prime \prime}$ N). The tower is surrounded by a hilly terrain covered with pine forest [41]. The average height above sea level is $180 \mathrm{~m}$. The average tree height $h$ is approximately $18 \mathrm{~m}$, and the topography height differences do not exceed the tree height [42]. The vegetation in a radius of $200 \mathrm{~m}$ from the tower is uniform; the buildings of the Hyytiälä scientific station and the small lake Kuivajärvi are $400 \mathrm{~m}$ away [43]. Multilevel continuous measurements of the concentrations of atmospheric gases and energy- and gas-exchange components in the atmosphere-biosphere system have been made on the tower since 1996 within the Fluxnet network [44]. Our analysis was done for the 2015-2016 winter period from November 5, 2015, to March 3, 2016, i.e., in the presence of a snow cover, when a stable boundary layer is most likely to exist.

We used data of micrometeorological measurements of the three components of wind speed and temperature at the heights $z=23,67$, and $125 \mathrm{~m}$ obtained by three-component ultrasonic anemometers (Solent Research 1012R2, Gill Instruments Ltd., Lymington, Hampshire, United Kingdom, at $23 \mathrm{~m}$ and USA-1, Metek GmbH, Elmshorn, Germany, at 67 and $125 \mathrm{~m}$ ). The primary measurement data at levels of 67 and $125 \mathrm{~m}$ were processed at a frequency of $10 \mathrm{~Hz}$. The mean wind speed and turbulent fluxes at these levels were calculated with averaging over 30 -min intervals. At the level of $23 \mathrm{~m}$, we used the calculated statistical turbu- lence parameters that are freely available in the SmartSMEAR database (https://avaa.tdata.fi/web/smart/ smear/). This database was also used for wind-speed values at $z=16.8$ and $33.6 \mathrm{~m}$. All data for unstable stratification $(\zeta<0)$ were excluded from the chosen dataset. Our calculations of turbulent exchange characteristics and the calculations presented on the site were based on the same software (EddyUH) [45]; i.e. the raw data prcessing was the same.

In the estimates given below, it is assumed that $D=$ $\frac{2}{3} h$ according to the value for a coniferous forest [46].

To estimate the height $H$ of a stable and neutral $\mathrm{ABL}$, we used time intervals where the turbulent characteristics are known near $z=23 \mathrm{~m}$ and at least at one of the levels $z=67 \mathrm{~m}$ or $z=125 \mathrm{~m}$. The ABL height was estimated in two ways using formulas (2): by a linear extrapolation of heat flux $F_{t}=\left\langle w^{\prime} T^{\prime}\right\rangle=\left(T_{0} / g\right) F_{b}$ (estimate of the ABL height $H_{F}$ ) and a linear extrapolation of the function $|\tau|^{2 / 3}$ (estimate of the height $H_{\tau}$ ). For the periods where the values of $F_{t}$ and $\tau$ are known at three levels, the required line was found by the least squares method. For coefficients of determination with $R^{2}<0.8$, the data were discarded. For the intervals where the fluxes are determined only at two levels, the line was drawn through two points; in this case, the data were assumed to be excluded from further analysis if the height estimate exceeded $2000 \mathrm{~m}$. In addition, the cases when the absolute values of fluxes increased with height were excluded. The final expression for determining the ABL height was $H=\left(H_{F}+H_{\tau}\right) / 2$. Here, the cases when the values of $H_{F}$ and $H_{\tau}$ significantly differed from one another were additionally excluded from the dataset: $\left|H_{F}-H_{\tau}\right|>\min \left(H_{F}, H_{\tau}\right) / 2$.

The average ABL height $\langle H\rangle$ under stable stratification for all chosen data was almost $280 \mathrm{~m}$. On some 30-min intervals, the estimate for $H$ turned out to be below the upper measurement level $z=125 \mathrm{~m}$ : these data were also excluded from analysis.

This sample allowed us to automatically exclude the certainly false data that contain unrealistic values of heat and momentum fluxes, which appeared to be associated with instrumental errors.

The vertical gradients of mean wind speed were calculated after approximating each profile by the logarithmic curve $\langle u\rangle=a+b \ln (c+z)$, where $a, b$, and $c$ are constants determined by the least squares method. The vertical gradient of the mean horizontal wind speed for each level $z_{k}$ was calculated as $d\langle u\rangle / d z=b /\left(c+z_{k}\right)$. It should be noted that the upper measurement level $z=$ $125 \mathrm{~m}$ was almost always in the upper ABL. For this level, a decrease in the dimensionless gradients $\phi_{m}(\xi)$ and $\phi_{m}^{\prime}\left(\xi^{\prime}\right)$ should be expected in comparison with the values given by universal functions (6) and (3). 

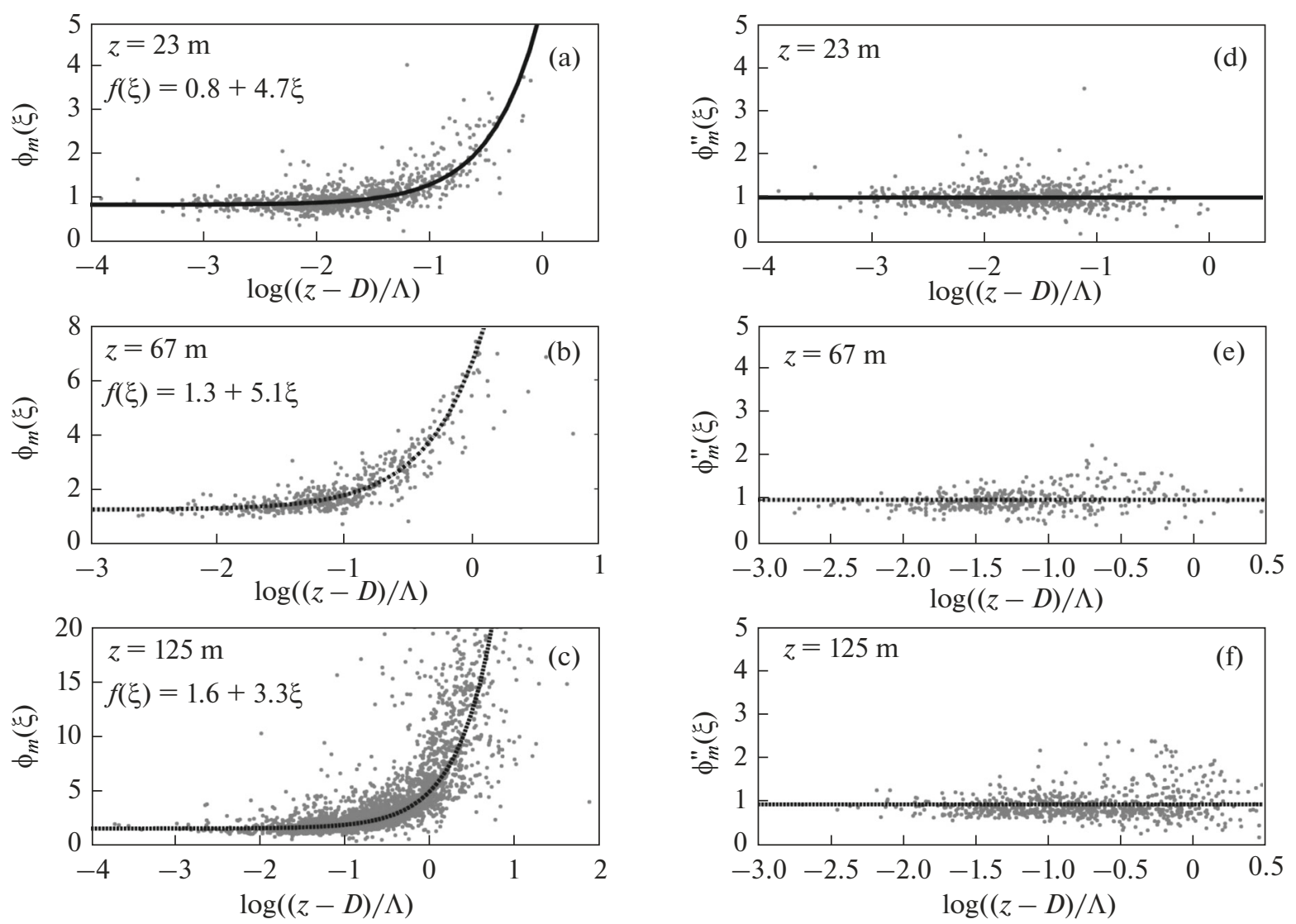

Fig. 1. Dimensionless velocity gradient $\phi_{m}$ normalized to the standard length scale $\xi=(z-D) / \Lambda$ at different levels. The dots denote observational data and the curves stand for the linear functions $f_{k}(\xi)=a_{k}+b_{k} \xi$ found by the least squares method (a, b, c). Velocity gradient normalized to the length scale $l_{t}^{m i x}: \phi_{m}^{\prime \prime}=(d\langle U\rangle / d z) l_{t}^{m i x} / u_{*}$. The dots denote observational data and the lines stand for mean values of $\phi_{m}^{\prime \prime}$ for the given level $z$.

\section{RESULTS}

\subsection{Verification of the Hypothesis that the Stratification Effect on the Profile of Mean Velocity over Inhomogeneous Landscape is Universal}

The dimensionless gradient of mean wind speed $\phi_{m}$ at three levels $\left(z_{1}=23 \mathrm{~m}, z_{2}=67 \mathrm{~m}\right.$, and $\left.z_{3}=125 \mathrm{~m}\right)$ as a function of the stability parameter $\xi=\kappa\left(z_{k}-D\right) / \Lambda$ is shown in Figs. 1a-1c. The dots denote measurement data and the solid curves were obtained by the least squares method: the dataset at each height matches its own linear dependence $f_{k}(\xi)=a_{k}+b_{k} \xi$. It can be seen that $a_{k}$ and $b_{k}$ are not universal and can significantly differ from the expected values $a=1$ and $b=C_{m}=5$.

The coefficient $a_{k}$ differs from unity at all levels. This means that the turbulence over the given surface is specific in comparison with the turbulence over landscapes with small roughness elements.

For the first two levels, coefficients $b_{k}$ are close to the value of $C_{m}=5$. The differences from this value are insignificant due to the large scatter of data, the inaccuracy in determining the mean velocity gradient, and the limited amount of data in the sample. This finding does not contradict the hypothesis proposed in Section 3 .

It should be noted that $b \approx 3<5$ for the upper level. This testifies that the turbulence scale increases in comparison with the scale determined by the local conditions of its generation and suppression by buoyancy forces. This effect can be explained by the presence of large eddies of nonlocal origin.

The empirical scale $l_{t}^{b}(z)$ (see formula (4)) was found by identifying the segments of neutral stratification with a stability parameter $\xi$ not exceeding the following thresholds for different levels: $\xi<10^{-2}(z=$ $\left.z_{1}=23 \mathrm{~m}\right), \xi<10^{-1.5}\left(z=z_{2}=67 \mathrm{~m}\right)$, and $\xi<10^{-1}$ $\left(z=z_{3}=125 \mathrm{~m}\right)$. The average value of was found for each measurement level:

$$
\begin{aligned}
& \left\langle l_{t}^{b}\left(z_{1}\right)\right\rangle / \kappa=14.6 \mathrm{~m} \quad(\text { at } z-D=11 \mathrm{~m}), \\
& \left\langle l_{t}^{b}\left(z_{2}\right)\right\rangle / \kappa=43.4 \mathrm{~m} \quad(\text { at } z-D=55 \mathrm{~m}), \\
& \left\langle l_{t}^{b}\left(z_{3}\right)\right\rangle / \kappa=74.9 \mathrm{~m} \quad(\text { at } z-D=113 \mathrm{~m}) .
\end{aligned}
$$

Vol. $54 \quad$ No. $5 \quad 2018$ 

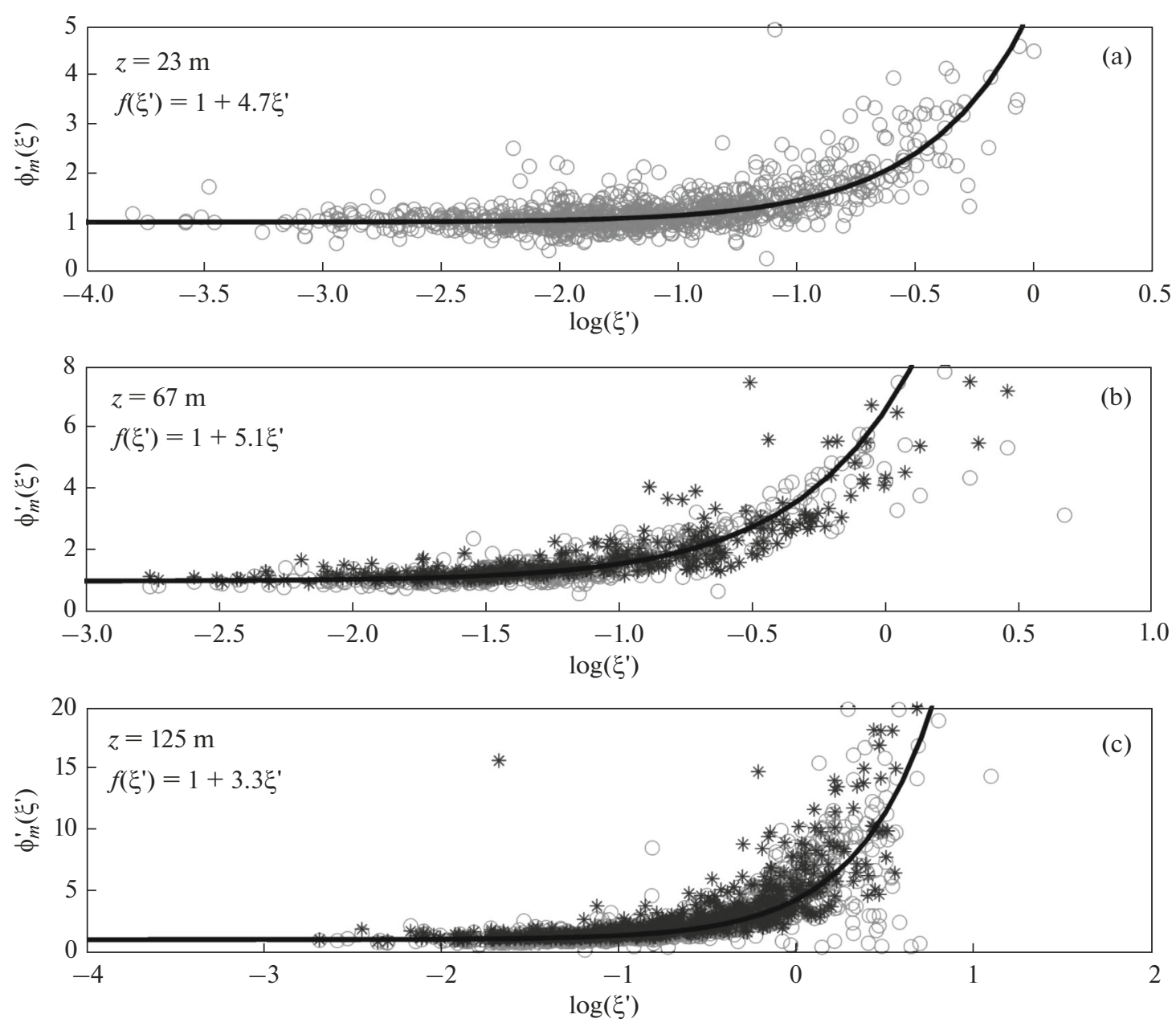

Fig. 2. Dimensionless velocity gradient $\phi_{m}^{\prime}$ normalized to empirical length scale $l_{t}^{b}$ at different levels. The light circles denotes the values of $\phi_{m}^{\prime}$ calculated from observational data, the asterisks stand for the values of $\phi_{m}^{\prime}$ estimated from the length scale $l_{t}^{m i x}$, and the curves indicate the linear functions $f_{k}\left(\xi^{\prime}\right)=a_{k}^{\prime}+b_{k}^{\prime} \xi^{\prime}$ found by the least squares method from observational data.

The empirical scale $l_{t}^{b}$ significantly differs from $\kappa(z-D)$, which is traditionally taken as a turbulence scale for neutral stratification according to formula (3). Here, this scale near the tree tops turns out to exceed $\kappa(z-D)$, which indicates the presence of relatively large eddies and is consistent with the conclusions drawn in [34, 36, 37].

Thus, topography and vegetation have a significant effect on the profile of mean wind speed in the ABL. This can be regarded as an effect that is independent of the influence of stratification. Primarily, the velocity gradient changes under nearly neutral conditions. Otherwise, the features of stable boundary layer over forest are similar to the features of the $A B L$ over a homogeneous surface with small roughness elements.

The dimensionless gradient $\phi_{m}^{\prime}$ calculated from observation data using normalization to the empirical length scale $l_{t}^{b}$ as a function of the stability parameter $\xi^{\prime}=l_{t}^{b} / \Lambda$ is shown in Figs. $2 \mathrm{a}-2 \mathrm{c}$ by light circles. The lines indicate the functions $f_{k}\left(\xi^{\prime}\right)=a_{k}^{\prime}+b_{k}^{\prime} \xi^{\prime}$ obtained by the least squares method. Due to the method used to construct the scale $l_{t}^{b}$, we have $a_{k}^{\prime} \approx 1$ and the coefficients $b_{k}^{\prime}$ coincide with $b_{k}$. Thus, this figure shows the dimensionless gradient of mean velocity in a form that coincides with this function over a homogeneous surface with small roughness elements (except for $125 \mathrm{~m}$, where $b_{k}=3.3$ ). It should be noted that the values of this function are highly scattered, which is especially significant for large values of $\xi$ ' and for the levels that are located away from the surface. The next section shows that this scatter is caused not only by instrumental errors and ABL nonstationarity, but also by the fact that there is no universal dependence of $\phi_{m}^{\prime}$ on the single dimensionless parameter $\xi^{\prime}$. 


\subsection{Normalization to the Turbulence Scale}

The dimensionless gradients $\phi_{m}^{\prime \prime}$ calculated from measurement data using the normalization to the scale $l_{t}^{\text {mix }}$ (formulas (8)-(13)) are shown in Figs. 1d-1f. For all measurement levels, the values of this function are in the vicinity of the lines $\phi_{m}^{\prime \prime} \approx$ const $\approx 1$. The mean values of $\phi_{m}^{\prime \prime}$ are shown by straight lines that are parallel to the abscissa axis. It should be noted that, in addition to reducing the dimensionless velocity gradient to a universal form, the above-described nondimensionalization decreases the number of vertically scattered points, i.e., reduces the uncertainty in calculating the gradient for a given value of $\xi^{\prime}$ due to the dependence on the dimensionless height $\sigma$.

The effect of the correction taking into account the $\mathrm{ABL}$ height and introduced with the help of the scale $l_{t}^{m i x}$ can be clearly demonstrated in Figs. $2 \mathrm{~b}$ and $2 \mathrm{c}$, which show not only the dimensionless velocity gradient $\phi_{m}^{\prime}$ calculated directly from observation data (light circles), but also its estimate obtained from formula (15) (asterisks). At the level $z_{1}$ (Fig. 2a), this estimate is not given because the scales at $\sigma=0$ are bound by the relationship $l_{t}^{m i x}=l_{t}^{b} /\left(1+C_{m} l_{t}^{b} / L\right)$ and the correction is not operational. Estimate (15) of the dimensionless gradient $\phi_{m}^{\prime}$ at all levels is within the data scatter. It can be seen that the scatter for this estimate at upper levels is comparable with the scatter of measured values of $\phi_{m}^{\prime}\left(\xi^{\prime}\right)$. The fact that $\phi_{m}^{\prime}\left(\xi^{\prime}\right)$ (or $\phi_{m}(z / \Lambda)$, which is analogous to it over a homogeneous surface) is not a universal function is caused by the fact that the measurements for equal values of the stability parameter $\xi$ ' can be made both near and away from the upper boundary of the ABL. In this case, the relative contribution of large eddies to the total momentum transfer along the vertical can vary significantly, which leads to a changed form of the mean wind velocity profile.

\section{CONCLUSIONS}

Micrometeorological measurements of temperature and wind speed in the ABL over a hilly surface covered with forest vegetation have been analyzed. The applicability of standard approaches to the construction of universal dependencies of the MoninObukhov similarity theory under stable and neutral stratification has been checked. An analysis of measurements under neutral stratification has shown that the approximation of the turbulent length scale by a linear dependence on height with a given displacement height is a very rough approximation.

An empirical base length scale depending on the local topography features and the underlying surface type has been proposed and calculated. This scale has been shown to allow the universal functions to be corrected for a stably stratified surface layer without sub- stantial modification, compared to the universal functions over a homogeneous surface with small roughness elements.

We have assumed that the nonlocal momentum transfer by large eddies is significant in the upper ABL. This assumption has been used to propose and verify a semiempirical nonuniversal parametrization of a fluxgradient relationship, taking into account the effect of topography, the features of vegetation cover, and the ABL height.

One disadvantage of this parameterization is that it requires the $A B L$ height estimation for calculating the dimensionless velocity gradient and using the results of the preliminary analysis of microveteorological measurements under neutral stratification to calculate the profile of the base length scale. To find generalizing regularities for surfaces that are similar in topography and vegetation cover, it is necessary to analyze the data obtained at different meteorological towers (we plan to do this in the future).

Knowing the distribution of the empirical length scale $l_{t}^{b}(z)$ over the Earth's surface or specifying this scale according to the surface types along with the roughness parameter $z_{0}$, one can introduce an adequate correction to calculate near-surface turbulent fluxes in atmospheric circulation models. This requires a verification of similar approximations of flux-gradient relationships for scalars (air temperature and humidity). In addition, the calculation of friction stress on the surface requires that the Ekman wind drift be taken into account. This is important for the parametrization of the land-atmosphere interaction under strong stability, when the height of lower reference levels in general atmospheric circulation models significantly exceeds the height of the constant flux layer and turns out to be comparable with the total ABL thickness. The authors of this paper plan to develop methods of this type and verify them against observational data in the nearest future.

In addition, there is a need to verify a similar approach for a convective-unstable surface layer, where the universal functions of dimensionless gradients are nonlinear and the replacement of the scale $z$ by a new empirical length scale cannot be easily justified.

\section{ACKNOWLEDGMENTS}

This study was conducted in the Scientific Research Computing Center of the Moscow State University and largely supported by the Russian Foundation for Basic Research, project no. 17-17-01210. The experimental measurements used in the study were supported by the Seventh Framework Program of the European Community, project no. 612642 (GHG-LAKE), and the Academy of Finland, project nos. 281196 (CarLAC), 307331 (Centre for Excellence), and 281255 (ICOSFINLAND). 


\section{REFERENCES}

1. A. S. Monin and A. M. Obukhov, "Main regularities of turbulent mixing in the atmospheric surface layer," Tr. Inst. Geofiz. Akad. Nauk SSSR 24, 163-187 (1954).

2. A. S. Monin and A. M. Yaglom, Statistical Fluid Mechanics (Gidrometeoizdat, St. Petersburg, 1992) [in Russian].

3. R. B. Stull, An Introduction to Boundary-Layer Meteorology (Kluwer, Boston, 1988).

4. Z. Sorbjan, Structure of the Atmospheric Boundary Layer (Prentice-Hall, Englewood Cliffs, NJ, 1989).

5. J. R. Garratt, The Atmospheric Boundary Layer (Cambridge University Press, Cambridge, 1992).

6. J. C. Kaimal and J. J. Finnigan, Atmospheric Boundary Layer Flows: Their Structure and 860 Measurements (Oxford University Press, New York-Oxford, 1994).

7. J. C. Wyngaard, Turbulence in the Atmosphere (Cambridge University Press, New York, 2010).

8. M. I. Mordukhovich and L. R. Tsvang, "Direct measurements of turbulent flows at two heights in the atmospheric surface layer," Izv. Akad. Nauk SSSR, Fiz. Atmos. Okeana 2 (8), 786-803 (1966).

9. Koprov B.M., Sokolov D.Yu. "Experimental study of the variability of heat flux in the atmospheric surface layer," Izv. Akad. Nauk SSSR, Fiz. Atmos. Okeana 11 (77), 743-747 (1975).

10. L. Mahrt, "Flux sampling errors for aircraft and towers," J. Atmos. Oceanic Technol. 15, 416-429 (1998).

11. G. N. Panin, G. Tetzlaff, and A. Raabe, "Inhomogeneity of the land surface and problem in parametrization of the surface fluxes in natural conditions," Theor. Appl. Clim. 60, 163-178 (1998).

12. Y. Malhi, D. D. Baldocchi, and P. G. Jarvis, "The carbon balance of tropical, temperate and boreal forests," Plant Cell Environ. 22, 715-740 (1999).

13. N. N. Vygodskaya, A. V. Varlagin, Yu. A. Kurbatova, A. V. Ol'chev, O. I. Panferov, F. A. Tatarinov, and N. V. Shalukhina, "Response of taiga ecosystems to extreme weather conditions and climate anomalies," Dokl. Biol. Sci. 429 (6), 571-574 (2009).

14. J. G. Canadell, H. A. Mooney, D. D. Baldocchi, J. A. Berry, J. R. Ehleringer, C. B. Field, S. T. Gower, D. Y. Hollinger, J. E. Hunt, R. B. Jackson, S. W. Running, G. R. Shaver, W. Steffen, S. E. Trumbore, R. Valentini, and B. Y. Bond, "Carbon metabolism of the terrestrial biosphere: A multitechnique approach for improved understanding," Ecosystems 3, 115-130 (2000).

15. M. O. Andreae and P. J. Crutzen, "Atmospheric aerosols: Biogeochemical sources and role in atmospheric chemistry," Science 276, 1052-1058 (1997).

16. H. Mayer and D. Schindler, "Forest meteorological fundamentals of storm damages in forests in connection with the extreme storm 'Lothar'," Allg. Forst- Jagdztg. 173, 200-208 (2002).

17. H. Bergström, H. Alfredsson, J. Arnqvist, I. Carlen, J. Fransson, E. Dellwik, H. Ganander, M. Mohr, and A. Segalini, Wind power in forests, Tech. Rep., 2013. http://elforsk.se//Raporter/?rid=13_09_.

18. J. R. Garratt, "Flux-profile relations above tall vegetation,” Q. J. R. Meteorol. Soc. 104, 199-211 (1978).
19. M. G. Villani, H. P. Schmid, J. L. Hutton, and C. S. Vogel, "Turbulence statistics measurements in a northern hardwood forest," Boundary-Layer Meteorol. 108, 343-364 (2003).

20. C. Q. Dias-Júnior, L. D. A. Sá, V. B. Pachêco, and C. M. de Souza, "Coherent structures detected in the unstable atmospheric surface layer above the Amazon forest," J. Wind Eng. Ind. Aerodyn. 115, 1-8 (2013).

21. D. Cava, U. Giostra, M. Siqueira, and G. Katul, "Organised motion and radiative perturbations in the nocturnal canopy sublayer above an even-aged pine-forest," Boundary-Layer Meteorol. 112, 129-157 (2004).

22. L. Mahrt, "The influence of nonstationarity on the turbulent flux-gradient relationship for stable stratification," Boundary-Layer Meteorol. 125, 245-264 (2007).

23. C. Q. Dias-Júnior, L. D. A. Sá, E. P. M. Filho, R. A. Santana, M. Mauder, and A. O. Manzi, "Turbulence regimes in the stable boundary layer above and within the Amazon forest," Agric. For. Meteorol. 233, 122-132 (2017).

24. S. Liu, H. Liu, M. Xu, M. Y. Leclerc, T. Zhu, C. Jin, Z. Hong, J. Li, and H. Liu, "Turbulence spectra and dissipation rates above and within a forest canopy," Boundary-Layer Meteorol. 98, 83-102 (2001).

25. B. D. Amiro, "Drag coefficients and turbulence spectra within three boreal forest canopies," Boundary-Layer Meteorol. 52, 227-246 (1990).

26. W. J. Massman and J. Weil, "An analytical one-dimensional second-order closure model of turbulence statistics and the Lagrangian time scale within and above plant canopies of arbitrary structure," Boundary-Layer Meteorol. 91, 81-107 (1999).

27. R. Leuning, "Estimation of scalar source/sink distributions in plant canopies using Lagrangian dispersion analysis: corrections for atmospheric stability and comparison with a multiplayer canopy model," BoundaryLayer Meteorol. 96, 293-314 (2000).

28. X. Lee, "Air motion within and above forest vegetation in non-ideal conditions," Forest Ecol. Manage. 135, 3-18 (2000). doi 10.1016/S0378-1127(00)00294-2

29. A. N. Ross, "Boundary-layer flow within and above a forest canopy of variable density," Q. J. R. Meteorol. Soc. 138, 1259-1272 (2012).

30. P. Hari and M. Kulmala, "Station for Measuring Ecosystem-Atmosphere Relations (SMEAR II)," Boreal Environ. Res. 10, 315-322 (2005).

31. J. A. Businger, J. C. Wyngaard, Y. Izumi, and E. F. Bradley, "Flux-profile relationships in the atmospheric surface layer," J. Atmos. Sci. 28 (2), 181-189 (1971).

32. F. T. M. Nieuwstadt, "The turbulent structure of the stable, nocturnal boundary layer," J. Atmos. Sci. 41 (14), 2202-2216 (1984).

33. A. A. Grachev, E. L. Andreas, C. W. Fairall, P. S. Guest, and P. O. G. Persson, "The critical Richardson number and limits of applicability of local similarity theory in the stable boundary layer," BoundaryLayer Meteorol. 147 (1), 51-82 (2013).

34. P. Cellier and Y. Brunet, "Flux-gradient relationships above tall plant canopies," Agric. For. Meteorol. 58, 93-117 (1992). 
35. A. V. Glazunov, "Numerical simulation of stably stratified turbulent flows over flat and urban surfaces," Izv., Atmos. Ocean. Phys. 50 (3), 236-245 (2014).

36. J. J. Finnigan, R. H. Shaw, and E. G. Patton, "Turbulence structure above a vegetation canopy," J. Fluid Mech. 637, 387-424 (2009).

37. Y. Brunet, J. J. Finnigan, and M. R. Raupach, "A wind tunnel study of air flow in waving wheat: Single-point velocity statistics," Boundary-Layer Meteorol. 70 (12), 95-132 (1994).

38. A. V. Glazunov, "Numerical simulation of stably stratified turbulent flows over an urban surface: Spectra and scales and parameterization of temperature and windvelocity profiles," Izv., Atmos. Ocean. Phys. 50 (4), 356-368 (2014).

39. A. M. Obukhov, "Turbulence scale distribution in arbitrary cross-section flows," Prikl. Mat. Mekh. 6 (2-3) 209-221 (1942).

40. A. V. Glazunov, "Numerical modeling of turbulent flows over an urban-type surface: Computations for neutral stratification," Izv., Atmos. Ocean. Phys. 50 (2), 134-142 (2014).

41. J. Heiskanen, M. Rautiainen, P. Stenberg, V. H. Vesanto, L. Korhonen, and T. Majasalmi, "Seasonal variation in MODIS LAI for a boreal forest area in Finland," Remote Sens. Environ. 126, 104-115 (2012).

42. P. Alekseychik, I. Mammarella, S. Launiainen, and Ü. Rannik, and T. Vesala, "Evolution of the nocturnal decoupled layer in a pine forest canopy," Agric. For. Meteorol. 174-175, 15-27 (2013).

43. I. Mammarella, A. Nordbo, Ü. Rannik, S. Haapanala, J. Levula, H. Laakso, A. Ojala, O. Peltola, J. Heiskanen, J. Pumpanen, and T. Vesala, "Carbon dioxide and energy fluxes over a small boreal lake in Southern Finland,” J. Geophys. Res.: Biogeosci. 120, 1296-1314 (2015). doi 10.1002/2014JG002873

44. D. Baldocchi, E. Falge, G. Lianhong, R. Olson, D. Hollinger, S. Running, P. Anthoni, Ch. Bernhofer, K. Davis, R. Evans, J. Fuentes, A. Goldstein, G. Katul, B. Law, X. Lee, Y. Malhi, T. Meyers, W. Munger, W. Oechel, U. Paw, K. Pilegaard, H. P. Schmid, R. Valentini, S. Verma, T. Vesala, K. Wilson, and S. Wofsy, "FLUXNET: A new tool to study the temporal and spatial variability of ecosystem-scale carbon dioxide, water vapor, and energy flux densities," Bull. Am. Meteorol. Soc. 82, 2415-2434 (2001).

45. I. Mammarella, O. Peltola, A. Nordbo, L. Järvi, and Ü. Rannik, "Quantifying the uncertainty of eddy covariance fluxes due to the use of different software packages and combinations of processing steps in two contrasting ecosystems," Atmos. Meas. Tech. 9, 49154933 (2016).

46. J. R. Garratt, "The atmospheric boundary layer," Earth Sci. Rev. 37 (1-2), 89-134 (1994).

Translated by V. Arutyunyan 